\title{
Accuracy of Lightweight Satellite Telemetry: a Case Study in the Iberian Peninsula
}

\author{
ALVARO SOUTULLO, ${ }^{1}$ Estación Biológica Terra Natura (CIBIO - Fundación Terra Natura), Universidad de Alicante, Apdo. correos 99 , \\ Alicante E-03080, Spain \\ LUIS CADAHÍA, Estación Biológica Terra Natura (CIBIO - Fundación Terra Natura), Universidad de Alicante, Apdo. correos 99, \\ Alicante E-03080, Spain \\ VICENTE URIOS, Estación Biológica Terra Natura (CIBIO - Fundación Terra Natura), Universidad de Alicante, Apdo. correos 99, \\ Alicante E-03080, Spain \\ MIGUEL FERRER, Departamento de Conservación de la Biodiversidad, Estación Biológica de Doñana, Consejo Superior de Investigaciones Cientificas, \\ Avda. de María Luisa s/n, Pabellón del Perú, Sevilla 41013, Spain \\ JUAN JOSÉ NEGRO, Departamento de Conservación de la Biodiversidad, Estación Biológica de Doñana, Consejo Superior de Investigaciones Científicas, \\ Avda. de María Luisa s/n, Pabellón del Perú, Sevilla 41013, Spain
}

\begin{abstract}
Here we provide the first assessment of the accuracy of lightweight satellite transmitters $(<80 \mathrm{~g})$ under actual operating conditions and the performance of the Argos system in southern Europe. To estimate transmitter accuracy we used transmitters equipped with a Global Positioning System (GPS) and compared the location estimates provided by Argos with the estimates provided by the GPS. Using the 68th percentile to define the accuracy of locations estimates, observed accuracy was $4 \mathrm{~km}$ for Location Class (LC) 1, $15 \mathrm{~km}$ for LC 0, $20 \mathrm{~km}$ for LC A, and $59 \mathrm{~km}$ for LC B, which is in line with estimates reported by other authors. Yet, the error of the remaining $32 \%$ of the data ranged between $4 \mathrm{~km}$ and $11 \mathrm{~km}, 15 \mathrm{~km}$ and $217 \mathrm{~km}, 20 \mathrm{~km}$ and $145 \mathrm{~km}$, and $59 \mathrm{~km}$ and $493 \mathrm{~km}$, respectively, suggesting that using the 68th percentile to estimate accuracies might give misleading confidence on the accuracy of location estimates. Using the 90th percentile is probably more appropriate. Less than $10 \%$ of the locations we obtained corresponded to the more accurate LCs (3, 2, and 1), with Argos failing to provide a position estimate in $45 \%$ of the attempts. The low number of high-quality location estimates is likely a consequence of the electromagnetic interference reported for our study area, rather than a defect of the Platform Transmitter Terminals (PTTs), which under good conditions of signal reception seem to be as reliable as heavier ones. The recent advent of lightweight GPS transmitters overrides most of these problems. Yet, whereas the smallest Argos-GPS PTTs weigh $30 \mathrm{~g}$, which restricts their use to animals weighting $>1,000 \mathrm{~g}$, conventional PTTs can be as small as $9.5 \mathrm{~g}$, allowing their use with animals weighting 250-300 g. (JOURNAL OF WILDLIFE MANAGEMENT 71(3):1010$1015 ; 2007)$
\end{abstract}

DOI: $10.2193 / 2006-042$

KEY WORDS accuracy, Argos, error, Europe, Platform Transmitter Terminal, satellite telemetry, satellite tracking.

Satellite telemetry has been used for tracking animal movements for more than 3 decades (Fancy et al. 1988, Britten et al. 1999). Although initially restricted to large vertebrates, technological improvements currently allow its use with animals as small as $300 \mathrm{~g}$ (Microwave Telemetry 2006). Despite these improvements, the reliability of lightweight $(<80 \mathrm{~g})$ transmitters (Platform Transmitter Terminals [PTTs]) has not yet been thoroughly explored. This is in part because lighter PTTs are becoming available every year (and thus there is virtually no time to assess their reliability), but also because an evaluation of the accuracy of Argos location estimates requires comparing them with the actual positions of the PTTs. This requires researchers to either compare the position with a few known positions, or use animals that can be easily located by other reliable methods. Consequently, most assessments of PTT accuracy and reliability are restricted to heavy transmitters $(>80 \mathrm{~g})$, attached to large vertebrates $(>10 \mathrm{~kg})$, and monitored for a few weeks (usually $<3$ months) within a relatively small area (e.g., Keating et al. 1991, Keating 1994, Vincent et al. 2002, White and Sjöberg 2002).

Since 2002 we have used satellite telemetry to track the juvenile dispersal of golden eagles (Aquila chrysaetos) and

${ }^{1}$ E-mail: a.soutullo@gmail.com
Bonelli's eagles (Hieraaetus fasciatus) in Spain (Cadahía et al. 2005; Soutullo et al. 2006a, b, c). In 2004 we fitted 5 individuals with newly released solar Argos-Global Positioning System (GPS) PTTs. These new PTTs use information from the GPS network to calculate their positions with an accuracy $<20 \mathrm{~m}$ (Microwave Telemetry 2006). Hence, these positions can be compared with the positions estimated by Argos based on the Doppler Effect (Argos estimates a position by measuring the Doppler shift of the PTT signal as a satellite passes over it; Fancy et al. 1988, Argos 1996, Britten et al. 1999, Hays et al. 2001), providing a means to evaluate the accuracy of Argos estimates.

Here we evaluate the accuracy with which Argos calculates the position of lightweight PTTs using data from the Iberian Peninsula. Most accuracy assessments have been conducted in North America (e.g., Keating et al. 1991, Keating 1994, Britten et al. 1999, Hatch et al. 2000), whereas published assessments from Europe are fairly scarce and mostly conducted with large vertebrates (e.g., Vincent et al. 2002, White and Sjöberg 2002). This is an important limitation for the evaluation of the reliability of results of studies conducted in Europe, where Argos performance may be well below that reported in other parts of the world, as there seems to be an important electromagnetic interference 


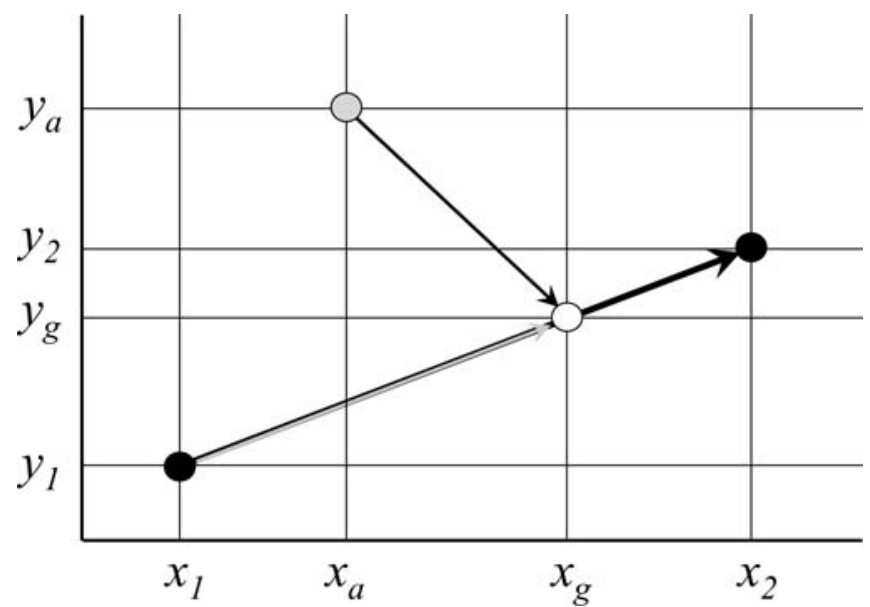

Figure 1. Estimation of the accuracy of the positions of lightweight satellite transmitters calculated using the Doppler Effect (Argos positions). We calculated the error as the distance between Argos positions $\left(x_{\mathrm{a}}, y_{\mathrm{a}}\right)$ and the true positions $\left(x_{\mathrm{g}}, y_{\mathrm{g}}\right)$ calculated on the basis of 2 consecutive Global Positioning System (GPS) locations $\left(x_{1}, y_{1}\right.$ and $\left.x_{2}, y_{2}\right)$. We considered as true positions those points within the straight line joining 2 consecutive GPS locations that were at a fraction $\left[\mathrm{a}=\left(t_{\mathrm{a}}-\mathrm{t}_{1}\right) /\left(t_{2}-t_{1}\right)\right]$ of the distance between the GPS locations, with $t_{\mathrm{a}}, t_{1}$ and $t_{2}$ being the time at which we obtained $\left(x_{\mathrm{a}}, y_{\mathrm{a}}\right),\left(x_{1}, y_{1}\right)$ and $\left(x_{2}, y_{2}\right)$, respectively.

with origin in southern Italy that is severely reducing (or even precluding) satellite reception throughout southern Europe and northern Africa (Microwave Telemetry 2005). This note constitutes the first assessment of the impact of that interference on the performance of studies conducted in the region using lightweight satellite telemetry.

\section{STUDY AREA}

We tagged birds in the Community of Valencia, eastern Spain, in nests located on rocky cliffs surrounded by Mediterranean scrublands. We then tracked birds' movements throughout most of the Iberian Peninsula, covering an area $>300,000 \mathrm{~km}^{2}$. The area encompassed a range of environments, including both high plateaus and mountain ranges, covered by deciduous and evergreen forests, scrublands, and cultivated areas. The climate was also extremely diverse, including areas dominated by Mediterranean, semiarid, subtropical, continental, oceanic, and alpine climates. This included warm to hot summers with mild to cool winters and annual precipitation averaging $600 \mathrm{~mm}$ in the Northern and Eastern Mediterranean coast, hot summers and mild to cool winters, with rainfall as low as $150 \mathrm{~mm}$ a year in the Southeast Mediterranean coast, and cold winters (depending mostly on altitude) and hot summers, with a relatively dry weather (precipitation 400$600 \mathrm{~mm} / \mathrm{yr}$ ) in the inner plateau.

\section{METHODS}

We analyzed the performance of $370-\mathrm{g}$ and $245-\mathrm{g}$ PTT100 Argos-GPS solar PTTs, all manufactured by Microwave Telemetry (Columbia, MD) and with a power output of $200 \mathrm{~mW}$. We attached the PTTs to 3 golden and 2 Bonelli's eagles while birds were still in the nest and tracked them between June 2004 and March 2005 (see Cadahía et al. [2005] and Soutullo et al. [2006b] for details on tagging and individuals). We programmed Argos-GPS PTTs to obtain hourly GPS fixes between 0600 hours and 2100 hours every day, and to transmit the data to Argos on a $\mathrm{SiV}^{\mathrm{TM}}$ (Satellite in View) schedule every third day (Microwave Telemetry 2006).

We downloaded positions estimated by Argos on the basis of the Doppler Effect from Argos' website using the DIAG command. For the GPS positions we used the PRV/A-DS command. For subsequent analyses we transformed all positions into Universal Transverse Mercator coordinates. We used the $\chi^{2}$ test to compare the proportion of locations in each location class (LC; a measure of estimates reliability) among PTTs, and to compare our results with those reported by Britten et al. (1999) for doves (Columba livia) and peregrines (Falco peregrinus).

We estimated accuracy of Argos positions as the minimum distance between positions estimated by Argos based on the Doppler Effect, and positions provided by the GPS. The time at which we obtained Argos and GPS positions rarely coincided because we obtained GPS positions every hour during the period PTTs were on, but Argos positions were only calculated when a satellite passed over a PTT. Therefore, we had to estimate the actual position of PTTs at the time their position was calculated by Argos. We calculated the magnitude of Argos' estimates error $(\varepsilon)$ as the distance $(\mathrm{km})$ between Argos positions $\left(x_{\mathrm{a}}, y_{\mathrm{a}}\right)$ and the actual positions $\left(x_{\mathrm{g}}, y_{\mathrm{g}}\right)$ calculated on the basis of 2 consecutive GPS locations $\left(x_{1}, y_{1}\right.$ and $x_{2}, y_{2}$; see Fig. 1). We calculated the actual (true) positions as those points within the straight line joining 2 consecutive GPS locations that are at a fraction $\left[\mathrm{a}=\left(t_{a}-t_{1}\right) /\left(t_{2}-t_{1}\right)\right]$ of the distance between the GPS locations, with $t_{a}, t_{1}$, and $t_{2}$ being the time at which we obtained the Argos position, the first GPS location, and the second GPS location, respectively. Hence, $x_{\mathrm{g}}=\mathrm{a} \times \cos (\arctan \mathrm{b})+x_{1}$, and $y_{\mathrm{g}}=\mathrm{a}$ $\times$ sine $(\arctan \mathrm{b})+y_{1}$, with $\mathrm{b}=\left(y_{2}-y_{1}\right) /\left(x_{2}-x_{1}\right)$, and thus, $\varepsilon=\sqrt{\left(x_{\mathrm{a}}-x_{\mathrm{g}}\right)^{2}+\left(y_{\mathrm{a}}-y_{\mathrm{g}}\right)^{2}}$. We calculated latitude and longitude errors as the distance between those positions in the latitudinal and longitudinal axes, respectively.

Every time a satellite passes over an active PTT Argos calculates 2 positions: the location (the estimate with better frequency continuity) and its image (Argos 1996, Britten et al. 1999). When the distance between the true position and the image provided by Argos was shorter than between the true position and Argos' location, we used the image to calculate the error of the position provided by Argos (7\% of the valid locations, $4 \%$ including $\mathrm{LC} \mathrm{Z}$ ).

We compared the accuracy of locations in different LCs with the Games-Howell multiple-comparisons test for unequal variances (Zar 1999). We also compared the distribution of errors' magnitude with a log-Normal distribution using the Kolmogorov-Smirnov test to fit the log-values of the observed errors to a Normal distribution. For each LC we used the Wilcoxon test to compare the magnitude of the latitude and longitude error of each location, and the Mann-Whitney test to compare the 
Table 1. Number of locations in each Argos' Location Class (LC) we obtained from 5 lightweight satellite transmitters (70 g and $45 \mathrm{~g}$ ) we deployed on 3 golden eagles and 2 Bonelli's eagles in Valencia, Spain, in June 2004.

\begin{tabular}{ccccccrrr}
\hline Identification no. & LC $\mathbf{3}$ & LC $\mathbf{2}$ & LC $\mathbf{1}$ & LC & LC A & LC B & LC Z & Total \\
\hline 49178 & 3 & 3 & 11 & 41 & 18 & 35 & 91 \\
49179 & 3 & 7 & 15 & 41 & 38 & 84 & 139 \\
49180 & 0 & 0 & 5 & 9 & 9 & 38 & 82 \\
49181 & 7 & 8 & 26 & 67 & 49 & 91 & 160 \\
49182 & 2 & 2 & 6 & 20 & 41 & 57 & 139 \\
Total & 15 & 20 & 63 & 178 & 155 & 305 & 611 \\
\% & 1.1 & 1.5 & 4.7 & 13.2 & 11.5 & 22.6 & 45.4 \\
\hline
\end{tabular}

magnitude of errors to the east of the true location with errors to the west, and errors to the north with errors to the south. Finally, for each LC we used linear and quadratic regressions between $t_{\mathrm{g}}$ and $\varepsilon$ to test for trends in accuracy as time differences between GPS and Argos' locations increased. We computed all statistical analyses using SPSS (SPSS Inc., Chicago, IL).

\section{RESULTS}

Forty-five percent of location attempts resulted in a failure of Argos to calculate a position. The number of locations provided by Argos decreased as the quality of the location class improved (Table 1). The proportion of locations in each LC differed among PTTs $\left(\chi^{2}=61.89, \mathrm{df}=24, P<0.001\right)$, and we received more $\left(\chi^{2}=546.68, \mathrm{df}=12, P<0.001\right)$ invalid locations ( $\mathrm{LC} \mathrm{Z}$ ) and less in LC 0 than Britten et al. (1999). Whereas the GPS provided 3,210 locations, Argos only calculated 736 locations from the 1,347 satellite passes recorded by the system. Of these, only 96 took place sometime between 2 consecutive GPS locations. We used these 96 to evaluate Argos locations' accuracy.

Due to the few LCs 3 and 2 locations received, we only evaluated the accuracy of LCs $1,0, A$, and B. The distribution of errors in the $4 \mathrm{LCs}$ was highly right-skewed, fitting a $\log$-Normal distribution (LC 1: $Z=0.821, n=7$, $P=0.511$; LC $0: Z=0.912, n=29, P=0.376$; LC A: $Z=$ $0.526, n=17, P=0.945$; LC B: $Z=0.651, n=41, P=$ $0.790)$. We did not observe differences in the accuracy of LCs 1 and 0 locations ( $\bar{x}$ difference $=0.563, P=0.096$ ), and LCs 0 and A locations ( $\bar{x}$ difference $=0.224, P=0.638$ ). Conversely, the accuracy of LC 1 locations was higher than that of LC A ( $\bar{x}$ difference $=0.787, P=0.025)$, with the accuracy of LC B locations being lower than observed for LCs $1(\bar{x}$ difference $=1.275, P<0.001), 0(\bar{x}$ difference $=$ $0.712, P<0.001)$, and A ( $\bar{x}$ difference $=0.488, P=0.053$ ).

In all LCs but LC 1 , the longitude error was larger than the latitude error (Fig. 2), with both longitude (LC 1: $Z=0.0, n$ $=7, P=1.0 ;$ LC $0: Z=0.524, n=29, P=0.621$; LC A: $Z=$ $0.586, n=17, P=0.601$; LC B: $Z=0.420, n=41, P=0.674)$ and latitude (LC 1: $Z=1.162, n=7, P=0.381 ; \mathrm{LC} 0: Z=$ $0.960, n=29, P=0.354$; LC A: $Z=0.878, n=17, P=0.417$; LC B: $Z=0.508, n=41, P=0.625$ ) errors being roughly symmetrically distributed with respect to the estimated true position. We did not observe a significant relationship (LC 1 linear: $F_{1,5}=0.22, P=0.655$; LC 1 quadratic: $F_{2,4}=0.16, P$ $=0.855$; LC 0 linear: $F_{1,27}=0.94, P=0.342$; LC 0 quadratic:
$F_{2,26}=0.75, P=0.481$; LC A linear: $F_{1,14}=0.54, P=0.476$; LC A quadratic: $F_{2,13}=1.0, P=0.395$; LC B linear: $F_{1,39}=$ $0.11, P=0.746$; LC B quadratic: $F_{2,38}=0.09, P=0.915$ ) between the estimated accuracy and the time GPS and Argos positions were calculated.

\section{DISCUSSION}

Less than $10 \%$ of the locations we obtained during the study period corresponded to the more accurate LCs. Actually, $45 \%$ of the times that location estimates were attempted, Argos was unable to provide an estimate. This is not surprising as lightweight PTTs are likely to show reduced accuracy compared to heavier units as a consequence of their larger susceptibility to shifts in temperature (Britten et al. 1999, Vincent et al. 2002). The situation is even worse if PTTs are attached to wide-ranging birds, which are constantly changing their velocity and altitude, and hence, increasing inaccuracy due to elevation and velocity error, and exposing the PTT to sudden changes in temperature and environmental conditions (Keating et al. 1991). However, the accuracy of the LCs we report here is similar to that reported by others under more controlled conditions or using heavier PTTs (e.g., Keating 1994, Brothers et al. 1998, Britten et al. 1999, Vincent et al. 2002, White and Sjöberg 2002), suggesting that under good conditions of signal reception, lightweight PTTs are as reliable as heavier ones. The low number of high-quality location estimates is likely a consequence of the electromagnetic interference reported for our study area (Microwave Telemetry 2005), rather than a defect of the PTTs. Actually, the frequency of occurrence of different LCs differed significantly among our PTTs and from those reported by Britten et al. (1999) for other lightweight PTTs. This interference is currently an important limitation to the use of satellite telemetry in southern Europe and northern Africa, highlighting the fact that marked variations in sampling frequencies may occur within and among studies (Keating et al. 1991) and, thus, the need for researchers to conduct their own assessments (Hays et al. 2001, Vincent et al. 2002).

A related issue worthy of mention is the way in which results are analyzed to estimate the accuracy of Argos locations. Both Argos and most authors (e.g., Argos 1996, Britten et al. 1999, Vincent et al. 2002, White and Sjöberg 2002) base their estimations of PTT accuracy on the 50$68 \%$ most accurate estimations (i.e., using as a benchmark the median or the 68th percentile of the distribution of 

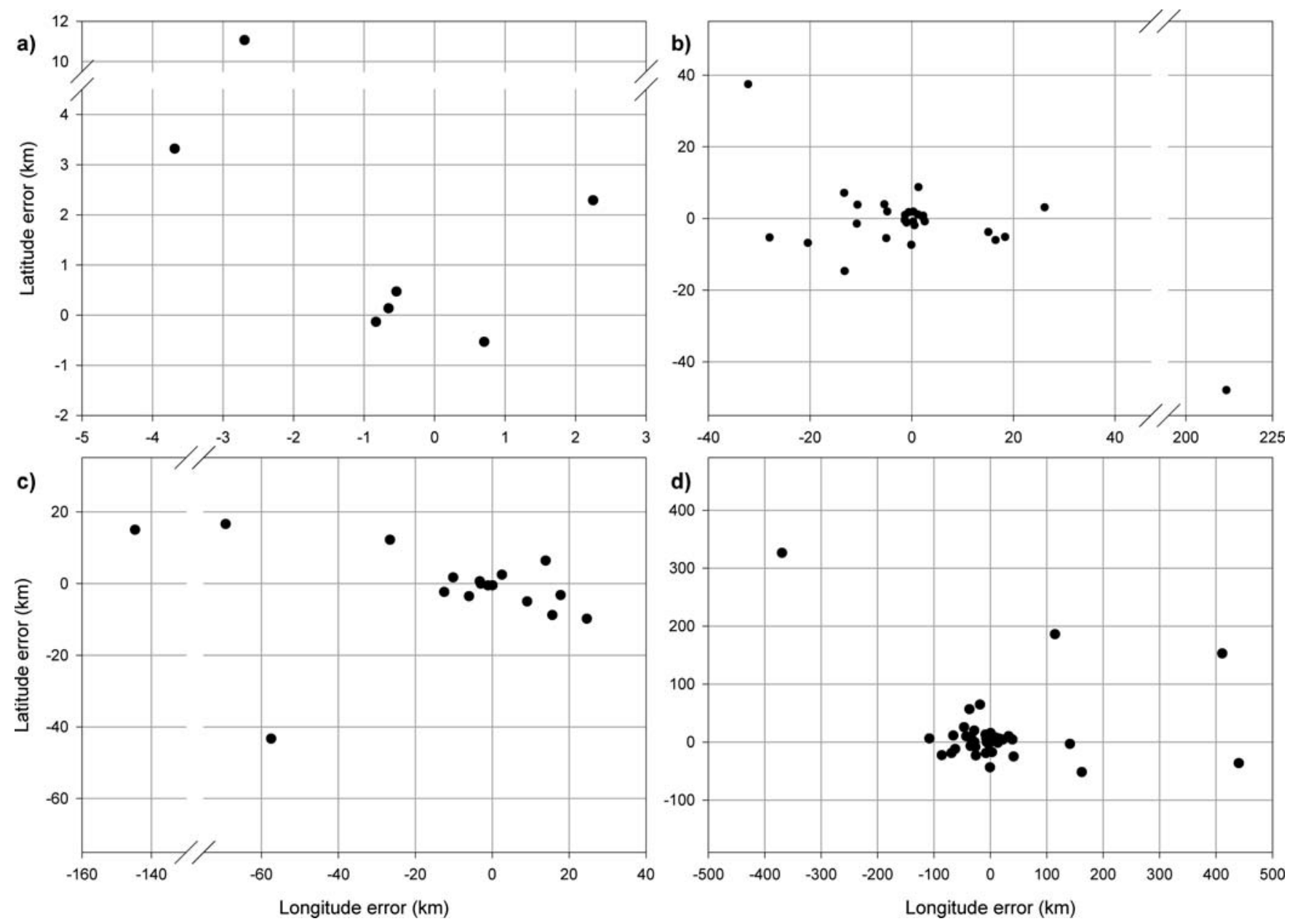

Figure 2. Latitude and longitude error ( $\mathrm{km}$ from the true location) of 5 lightweight satellite transmitters $(70 \mathrm{~g}$ and $45 \mathrm{~g})$ we deployed on 3 golden eagles and 2 Bonelli's eagles in Valencia, Spain, in June 2004) a) Location Class (LC) 1 locations (latitude error = longitude error); b) LC 0 locations (latitude error < longitude error); c) LC A locations (latitude error < longitude error); and d) LC B locations (latitude error < longitude error). Negative values indicate south and west.

errors). Given the highly right-skewed distribution of errors, this means that there is roughly a $32 \%$ probability of obtaining locations that are much further away from the true position than stated. Using the 68th percentile to define the accuracy with which locations in different LCs are estimated, we would conclude from our data that locations in LC 1 have an accuracy of $4 \mathrm{~km}$, in LC 0 have an accuracy of $15 \mathrm{~km}$, in LC A have an accuracy of $20 \mathrm{~km}$, and in LC B have an accuracy of $59 \mathrm{~km}$. Yet, it also means that there is roughly a $32 \%$ probability of obtaining locations that are between $4 \mathrm{~km}$ and $11 \mathrm{~km}, 15 \mathrm{~km}$ and $217 \mathrm{~km}, 20 \mathrm{~km}$ and $145 \mathrm{~km}$, and $59 \mathrm{~km}$ and $493 \mathrm{~km}$ away from the true position, respectively (Table 2). Thus, we suggest using a value closer to the 90th percentile to report the accuracy of different LCs. This is more conservative than using the 68th percentile, which may give researchers a misleading perception of the reliability of their data. In any case, accuracy can be enhanced if researchers use appropriate filtering procedures. Most of these procedures use information about species behavior to remove implausible locations (e.g., Hays et al. 2001, Potavov and Dubinin 2005), and we strongly advocate their use when dealing with Argos data.
Argos performance is not uniform throughout the globe and average accuracy will probably differ from study to study. Thus, when using conventional lightweight PTTs, researchers need to consider that there is usually a trade-off between accuracy and sample size. In accordance with Vincent et al. (2002) and White and Sjöberg (2002), we suggest considering using LC A (and LC 0) locations, as they provide a large amount of relatively accurate data. When aiming at tracking gross movements, even LC B can be useful if sample size, rather than accuracy, is a problem and filtering procedures are used (e.g., Douglas 2000, Potapov and Dubinin 2005). Conversely, by using ArgosGPS PTTs, research questions requiring accuracy of $<20 \mathrm{~m}$ can be addressed straightforwardly (Microwave Telemetry 2006). Moreover, not only are Argos-GPS PTTs just about $20 \%$ more expensive than conventional ones (Microwave Telemetry 2006), but also sample size can be much larger than that obtained using conventional PTTs, as the frequency with which locations are calculated can be set at will (e.g., every sec), enabling very detailed tracking of animal movements (e.g., Weimerskirch et al. 2002). Furthermore, as location frequency and accuracy is inde- 
Table 2. Accuracy of Argos' estimates of the positions of 5 lightweight satellite transmitters $(70 \mathrm{~g}$ and $45 \mathrm{~g})$ we deployed on 3 golden eagles and 2 Bonelli's eagles in Valencia, Spain, in June 2004. The values indicate the distance $(\mathrm{km})$ to the true position estimated by the Global Positioning System.

\begin{tabular}{|c|c|c|c|c|c|c|}
\hline & $\mathrm{LC}^{\mathrm{a}} 3$ & LC 2 & LC 1 & LC 0 & LC A & LC B \\
\hline $\bar{x}$ & 0.3 & 1.3 & 3.2 & 17.6 & 26.3 & 76.1 \\
\hline SE & & & 1.5 & 7.4 & 9.1 & 18.4 \\
\hline SD & & & 4.0 & 39.9 & 37.6 & 117.8 \\
\hline Median & 0.3 & 1.3 & 0.9 & 7.4 & 12.7 & 35.0 \\
\hline 68th percentile & & & 4.0 & 15.3 & 20.0 & 59.1 \\
\hline 90th percentile & & & 11.4 & 28.5 & 86.7 & 208.8 \\
\hline 95th percentile & & & 11.4 & 133.2 & 145.5 & 441.4 \\
\hline Min. & 0.3 & 1.3 & 0.7 & 0.9 & 0.5 & 1.1 \\
\hline Max. & 0.3 & 1.3 & 11.4 & 217.1 & 145.6 & 493.1 \\
\hline Skewness & & & 1.8 & 4.8 & 2.4 & 2.7 \\
\hline Kurtosis & & & 3.4 & 24.3 & 6.1 & 6.8 \\
\hline$N$ & 1 & 1 & 7 & 29 & 17 & 41 \\
\hline
\end{tabular}

${ }^{\mathrm{a}} \mathrm{LC}=$ location class.

pendent of the quality and frequency of valid Argos locations, GPS telemetry is especially suitable for Europe and other areas where interferences hinder the performance of the Argos system. Yet, whereas the smallest Argos-GPS PTTs weigh $30 \mathrm{~g}$, conventional PTTs can be as small as 9.5 $\mathrm{g}$ (Microwave Telemetry 2006). This implies that researchers can track only about 500 bird species using Argos-GPS PTTs, whereas they can study 3 times as many using conventional satellite transmitters (figures derived from Blackburn and Gaston 1994, assuming PTT wt does not represent $>3 \%$ of the animal wt, as suggested by Kenward 2001). Therefore, conventional satellite telemetry is still the best technique available for tracking long- to medium-range movements of animals between $300 \mathrm{~g}$ and 1,000 g.

\section{MANAGEMENT IMPLICATIONS}

Accuracy of lightweight satellite transmitters is comparable to that reported for heavier units, enabling tracking long- to medium-range movements of animals weighing $>300 \mathrm{~g}$. Lightweight satellite telemetry is, thus, a powerful tool to gather information on home range size and use, the temporal pattern of daily activities, and the location of temporary settlements, corridors, migration routes and stopover sites, all of which are of primary interest for wildlife management and conservation. However, it is inappropriate for detailed ( $<1,000 \mathrm{~m}$ accuracy) descriptions of habitat use, or for studies that require a large number of daily locations, or that locations are obtained at precise times of the day or at regular intervals. Also, their usefulness can be severely reduced where the signal is degraded by landscape features or electromagnetic interference, as in southern Europe. Where possible, GPS transmitters are to be preferred, as they are 2 orders of magnitude more accurate, can be programmed to obtain locations at preset times or regular intervals, and they do not depend on signal reception for the estimation of their position.

\section{ACKNOWLEDGMENTS}

Thanks are due to the Consellería de Territori i Habitatge of the Generalitat Valenciana (J. Jiménez, P. Mateache, A. Izquierdo, and A. García i Sanz) and the Spanish Ministerio de Medio Ambiente (V. García Matarranz, P. García Domínguez) for partial funding, permission to get access to the nests, and invaluable field assistance. The Terra Natura Foundation funded this project. We are very grateful to Y. Canzani for mathematical assistance, to K. Steenhof for critically reviewing the manuscript, and to J. Carroll, S. A. Hatch, and an anonymous referee for constructive suggestions. L. Cadahía is holder of a scholarship from the Spanish Ministerio de Educación y Ciencia (reference AP20011444). This paper is part of A. Soutullo's Dissertation at the Universidad de Alicante.

\section{LITERATURE CITED}

Argos. 1996. User's manual. CLS/Service Argos, Toulouse, France.

Blackburn, T. M., and K. J. Gaston. 1994. The distribution of body sizes of the world's bird species. Oikos 70:127-130.

Britten, M. W., P. L. Kennedy, and S. Ambrose. 1999. Performance and accuracy evaluation of small satellite transmitters. Journal of Wildlife Management 63:1349-1358.

Brothers, N., R. Gales, A. Hedd, and G. Robertson. 1998. Foraging movements of the shy albatross Diomedea cauta breeding in Australia: implications for interactions with longline fisheries. Ibis 140:446-457.

Cadahía, L., V. Urios, and J. J. Negro. 2005. Survival and movements of satellite tracked Bonelli's Eagles during their first winter. Ibis 147: 415-419.

Douglas, D. C. 2000. PC-SAS Argos Filter V5.0 Software Documentation. United States Geological Survey, Alaska Biological Science Center, Anchorage, USA.

Fancy, S. G., L F. Pank, D. C. Douglas, C. H. Curby, G. W. Garner, S. C. Amstrup, and W. L. Regelin. 1988. Satellite telemetry: a new tool for wildlife research and management. U.S. Fish and Wildlife Service Resource Publication 172, Washington, D.C., USA.

Hatch, S. A., P. M. Myers, D. M. Mucahy, and D. C. Douglas. 2000. Performance of implantable satellite transmitters in diving seabirds. Waterbirds 23:84-94.

Hays, G. C., S. Åkesson, B. J. Godley, P. Luschi, and P. Santidrian. 2001. The implications of location accuracy for the interpretation of satellitetracking data. Animal Behaviour 61:1035-1040.

Keating, K. A. 1994. An alternative index of satellite telemetry location error. Journal of Wildlife Management 58:414-421.

Keating, K. A., W. G. Brewster, and C. H. Key. 1991. Satellite telemetry: performance of animal-tracking systems. Journal of Wildlife Management 55:160-171.

Kenward, R. E. 2001. A manual for wildlife radio tagging. Academic Press, London, United Kingdom.

Microwave Telemetry. 2005. Argos performance in Europe. Tracker News 6(2):8. 
Microwave Telemetry. 2006. Microwave Telemetry home page. < http:// www.microwavetelemetry.com>. Accessed 1 Feb 2006.

Potapov, E., and M. Dubinin. 2005. 'Argos-tools' manual. The Falcon Research Institute, National Avian Research Center, Carmarthen, United Kingdom.

Soutullo, A., V. Urios, and M. Ferrer. 2006a. How far away in an hour? Daily movements of juvenile golden eagles (Aquila chrysaetos) tracked with satellite telemetry. Journal of Ornithology 147:69-72.

Soutullo, A., V. Urios, M. Ferrer, and S. G. Peñarrubia. 2006b. Dispersal of golden eagles Aquila chrysaetos during their first year of life. Bird Study $53: 258-264$

Soutullo, A., V. Urios, M. Ferrer, and S. G. Peñarrubia. 2006c. Post- fledging behaviour in golden eagles: onset of the juvenile dispersal and progressive distancing from the nest. Ibis 148:307-312.

Vincent, C., B. J. McConnel, M. A. Fedak, and V. Ridoux. 2002. Assessment of Argos location accuracy from satellite tags deployed on captive grey seals. Marine Mammal Science 18:301-322.

Weimerskirch, H., F. Bonadonna, F. Bailleul, G. Mabille, G. Dell'Omo, and H. Lipp. 2002. GPS tracking of foraging albatrosses. Science 295:1259.

White, N. E., and M. Sjöberg. 2002. Accuracy of satellite positions from free-ranging grey seals using Argos. Polar Biology 25:629-631.

Zar, J. H. 1999. Biostatistical analysis. Fourth edition. Prentice Hall, New Jersey, USA.

Associate Editor: Carroll. 